\title{
Mammalian sterile 20-like kinase 1 expression and its prognostic significance in patients with breast cancer
}

\author{
XIAO-YAN LIN ${ }^{1 *}$, FENG-FENG CAI $^{1^{*}}$, MING-HONG WANG $^{2 *}$, XIN PAN $^{3}$,

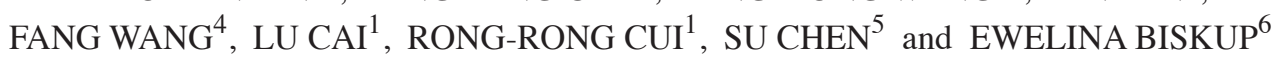 \\ ${ }^{1}$ Department of Breast Surgery, Yangpu Hospital, Tongji University School of Medicine, Shanghai 200090; \\ ${ }^{2}$ Department of Cardiology, Eastern Hepatobiliary Surgery Hospital, Second Military Medical University, \\ Shanghai 200438; ${ }^{3}$ Central Laboratory; ${ }^{4}$ Department of Pharmacy, Yangpu Hospital, Tongji University \\ School of Medicine, Shanghai 200090; ${ }^{5}$ Department of Molecular and Cellular Biology, School of \\ Forensic Sciences, Xi'an Jiao Tong University Health Science Center, Xi'an, Shaanxi 710061, \\ P.R. China; ${ }^{6}$ Department of Internal Medicine, University Hospital of Basel, Basel 4031, Switzerland
}

Received February 28, 2016; Accepted July 21, 2017

DOI: $10.3892 / 01.2017 .6852$

\begin{abstract}
Mammalian sterile 20-like kinase 1 (Mst1) is a major inhibitor of cell proliferation, and is involved in apoptosis, oncogenesis and organ growth via its ubiquitously encoded serine threonine kinase. Previous studies have demonstrated that Mst1 has a tumor suppressor function in human breast cancer. Mst1 deletion or mutation is associated with tumorigenesis, whereas Mst1 overexpression leads to tumor cell apoptosis and decreases proliferation of tumor cells. Our previous study reported the tumor suppressive function of Mst1, and debated Mst1 as a prognostic factor in human breast cancer. In the present study, Mst1 levels were measured in the plasma of patients in order to elucidate their association with overall and disease-free survival. The results of the present study indicated that Mstl is a strong prognostic and predictive factor in human breast cancer and a promising anticancer target.
\end{abstract}

Correspondence to: Dr Feng-Feng Cai, Department of Breast Surgery, Yangpu Hospital, Tongji University School of Medicine, 450 Teng-Yue Road, Shanghai 200090, P.R. China

E-mail: caifengfeng2013@163.com

Dr Xin Pan, Central Laboratory, Yangpu Hospital, Tongji University School of Medicine, 450 Teng-Yue Road, Shanghai 200090, P.R. China

E-mail: xinpanpx@163.com

*Contributed equally

Key words: mammalian sterile 20-like kinase 1, breast cancer, prognosis, tumor suppressor gene, biomarker

\section{Introduction}

Breast cancer is the most common type of cancer in women worldwide and the second most common type of cancer overall (1-3). Despite advances being made in therapeutic and diagnostic study, only a fraction of treatment strategies are individualized, with treatment based not only on a careful risk assessment for each patient, but on specific clinicopathological features of the breast cancer they suffer from (4-6). There are a number of established predictive factors in breast cancer, the majority of which are also therapeutic targets [including estrogen receptor, progesterone receptor or human epidermal growth factor receptor 2 (Her2)] (7). Therapeutic approaches are primarily based on clinicopathological variables, including tumor size, lymph node stage, histological grade, type and lymphovascular invasion (8). However, there is a limited choice of prognostic factors, which are essential for decision-making, since these predict patient outcomes irrespective of treatment (9). Furthermore, breast cancer is one of the most heterogeneous diseases. There are a growing number of aged patients ( $>75$ years) with breast cancer, and therefore there is an urgent requirement for personalized therapies in order to avoid over- or under-treatment (10-12). Multi-parameter gene expression analyses are evolving and progressively suggesting promising markers (13-15). Thus, it is important to study and propose methods for an effective and efficient quantification of specific gene expression status (16). At the same time, evidence of statistical association between the gene expression and overall survival (OS) and disease-free survival (DFS) are required for designing antitumor management strategies and prognosis evaluation $(17,18)$.

Mammalian sterile 20-like kinase 1 (Mst1), alternatively termed serine threonine kinase 4 (STK4), has been known for decades, but regained significant attention when its role as a tumor suppression gene in various entities of human cancers was reported (19-22). Mst1 is a serine/threonine protein kinase, which builds a complex with Mst2. Mst1/Mst2 are activated by the phosphorylation of $\mathrm{Thr}^{183}$ and $\mathrm{Thr}^{180}$, which leads to a 
feedback stimulation that regulates oxidant levels through a number of mechanisms (23). One of them is the regulation of cellular redox state (23). This specific mechanism may represent a tumor suppressor function of Mst1/2 (23,24). Mst1/2 kinases also affect immune cell activation, proliferation, adhesion, migration, growth and apoptotic pathways, as they are essential for the Hippo signaling pathway (25). Loss of Mst1 results in hyper-proliferation and tumorigenesis (25). In addition, the Hippo pathway interacts with other signaling pathways, including Wnt and Notch pathways, known for their crucial roles in tumor pathogenesis $(26,27)$. Finally, the pro-apoptotic function of Mst1 is also associated with pleckstrin homologydomain and leucine-rich repeat protein phosphatases (28). These two proteins synergize to achieve a greater potential of apoptosis (28). Phosphoinositide 3-kinase/protein kinase Bacts as an inhibitor of Mst1 through phosphorylation of threonine 120 (29).

Our previous study, for the first time, presented data that supported the function of Mst1 as a prognostic factor in human breast cancer, using immunostaining as the Mst1-identification method (30). This study demonstrated that Mst1-positive patients had a significantly improved OS compared with Mst1-negative patients, and that Mst1 is an independent prognostic factor in breast cancer. In the present study, ELISA was performed to quantify Mst1 concentration in the serum of patients with breast cancer. The methodological concept facilitates a direct translation into the clinic, as it is easy, feasible, exact and less biased than immunohistological estimations of the amount of Mst1 in tumor cells. In addition, the sampling method is incomparably more attractive for the daily routine and comfort of patients. The present study demonstrated the prognostic significance of Mstl expression for the rates of OS and DFS. The results of the present study revealed the tumor suppressive function of Mst1, and confirmed Mst1 as an independent prognostic factor in human breast cancer.

\section{Materials and methods}

Ethical approval. The present study was performed at the Central Laboratory and Department of Breast Surgery, Yangpu Hospital, Tongji University School of Medicine (Shanghai, China), and was approved by the local institutional review board. Written consent forms were collected from all patients involved in the present study. The ethics review board of Tongji University School of Medicine approved the study design.

Study population. Blood samples used in the present study were collected between January 2005 and December 2006 in the Department of Breast Surgery, Yangpu Hospital, Tongji University School of Medicine. In total, 98 women were included in the study, since they completed the entire period of follow-up. All blood samples were taken prior to any surgical interventions or antitumor treatment. Data of patients, including age, tumor size, tumor stage, histological grade, node status, histological type, molecular subtypes, hormone receptor status and Her2 status were obtained from the pathological reports. Table I describes the baseline demographics of the study population. The distribution of tumor grades and receptor status were representative. The majority of the patients presented with carcinoma of a ductal type with luminal subtype, grade 2 . All patients, the median age was 52 and the age range of patients was 35-73, were Chinese females and were followed until mortality or the end of the follow-up period of 98 months.

Indirect ELISA detection of Mst1 in the plasma of patients. ELISA detection was used as an efficient and effective method in order to assess the expression level of Mst1 in plasma samples and associated them with the survival of patients. A total of 98 human plasma samples were assayed by ELISA using Mst1/STK4 (C-term) antibodies. The Mst1/STK4 purified protein was included in the assay system as a positive control for specificity and sensitivity, as well as to create a calibration curve. Each assay was repeated three times.

In brief, flat-bottom 96-well Costar plates were coated with $100 \mu 1$ per well of rabbit polyclonal antibody specific for human Mst1/STK4 (cat. no. 3682; Cell Signaling Technology, Inc., Danvers, MA, USA) at a concentration of $1 \mu \mathrm{g} / \mathrm{ml}$ in carbonate buffer (15 mM Na${ }_{2} \mathrm{CO}_{3}, 35 \mathrm{mM} \mathrm{NaHCO}$, pH 9.6) as previously described (31). Following an overnight incubation at $4^{\circ} \mathrm{C}$, the plates were washed three times with PBS-Tween-20 (PBST; $1.47 \mathrm{mmol} / 1 \mathrm{KH}_{2} \mathrm{PO}_{4}, 8.10 \mathrm{mmol} / \mathrm{l}$ $\mathrm{Na}_{2} \mathrm{HPO}_{4}, 136.89 \mathrm{mmol} / 1 \mathrm{NaCl}, 2.68 \mathrm{mmol} / \mathrm{l} \mathrm{KCl}, 0.05 \%$ Tween 20), blocked with blocking buffer [1\% bovine serum albumin (BSA; w/v; cat. no. A3858; Sigma-Aldrich; Merck $\mathrm{KGaA}$, Darmstadt, Germany) in PBST] for $1 \mathrm{~h}$ at $37^{\circ} \mathrm{C}$, followed by washing with PBST three times. Subsequently, the clinical plasma samples were diluted 5-fold (1:5) in sample diluent (1\% BSA in PBST). Pre-diluted samples (100 $\mu \mathrm{l})$ was added into micro ELISA plate wells. PBS served as a blank control. Following incubation for $2 \mathrm{~h}$ at $37^{\circ} \mathrm{C}$, the wells were again washed and filled with $100 \mu \mathrm{l}$ of a 1:10,000 dilution of horseradish peroxidase-conjugated goat anti-rabbit antibody (cat. no. 1662408EDU; Bio-Rad Laboratories, Inc., Hercules, CA, USA). Following incubation for $1 \mathrm{~h}$ at $37^{\circ} \mathrm{C}$, plates were again washed with PBST, and wells were filled with $100 \mu 1$ 3,3',5,5'-tetramethylbenzidine substrate solution and incubated for $15 \mathrm{~min}$ at room temperature in the dark. The reaction was stopped by adding $50 \mu \mathrm{l} 2 \mathrm{~mol} / \mathrm{H} \mathrm{H}_{2} \mathrm{SO}_{4}$ per well. The optical density of each well was measured at a wavelength of $450 \mathrm{~nm}$ in the ELISA plate reader. Calibration curves were generated with $\log _{10}$ Mst1/STK4 purified protein concentrations plotted along the $\mathrm{x}$-axis. If the detected values were higher than average, plasma samples were judged as positive.

Statistical analysis. Data were analyzed by SPSS standard version 20.0 (IBM SPSS, Armonk, NY, USA). The Kaplan-Meier method was used to estimate OS and DFS. A log rank test was used to compare the survival curves. Multivariate analysis was performed by Cox proportional hazards model. OS was calculated from the date of diagnosis to the date of mortality or the last follow-up. DFS was calculated from the date of surgery to the date of disease relapse. The differences between groups were analyzed using an unpaired two-tailed Student's t-test. All P-values were two-tailed. $\mathrm{P}<0.05$ was considered to indicate a statistically significant difference. 
Table I. Patients and tumor characteristics.

\begin{tabular}{|c|c|}
\hline Variables & Patients, n (\%) \\
\hline \multicolumn{2}{|l|}{ Age, years } \\
\hline$<50$ & $27(27.6)$ \\
\hline$\geq 50$ & $71(72.4)$ \\
\hline \multicolumn{2}{|c|}{ Tumor size, $\mathrm{cm}$} \\
\hline$<2$ & $42(42.9)$ \\
\hline$\geq 2$ & $56(57.1)$ \\
\hline \multicolumn{2}{|c|}{ Tumor stage } \\
\hline $\mathrm{T} 1$ & $27(27.6)$ \\
\hline $\mathrm{T} 2$ & $52(53.1)$ \\
\hline $\mathrm{T} 3$ & $19(19.3)$ \\
\hline \multicolumn{2}{|c|}{ Histological grade } \\
\hline G1 & $5(5.1)$ \\
\hline G2 & $78(79.6)$ \\
\hline G3 & $15(15.3)$ \\
\hline \multicolumn{2}{|c|}{ Lymph node status } \\
\hline Negative & $57(58.2)$ \\
\hline Positive & $41(41.8)$ \\
\hline \multicolumn{2}{|c|}{ Histological type } \\
\hline Ductal & $87(88.8)$ \\
\hline Others & $11(11.2)$ \\
\hline \multicolumn{2}{|c|}{ Molecular subtypes } \\
\hline Luminal & $68(30.6)$ \\
\hline Others & $30(69.4)$ \\
\hline \multicolumn{2}{|l|}{ HR status } \\
\hline Negative & $30(30.6)$ \\
\hline Positive & $68(69.4)$ \\
\hline \multicolumn{2}{|l|}{ Her2 status } \\
\hline Negative & $71(72.4)$ \\
\hline Positive & $27(27.6)$ \\
\hline \multicolumn{2}{|l|}{ Mst1 status } \\
\hline Negative & $13(13.3)$ \\
\hline Positive & $85(86.7)$ \\
\hline
\end{tabular}

Mst1, mammalian sterile 20-like kinase 1; Her2, human epidermal growth factor receptor 2; HR, hormone receptor.

\section{Results}

Patient characteristics. Characteristics of the 98 patients enrolled in the present study are summarized in Table I. No patients succumbed and no patients withdrew during the study period. The follow-up time was 98 months.

Mst1 levels in patients with breast cancer. A total of 98 human plasma samples were assayed by ELISA. The average Mst1 value of 98 human plasma samples was $1.8 \mu \mathrm{g} / \mathrm{ml}$. Profiles of immunoglobulin IgG antibodies against human plasma Mst1 antigens were estimated by indirect ELISA (Fig. 1). The average concentration of $1.8 \mu \mathrm{g} / \mathrm{ml}$ was used to discriminate the status of Mst1-positivevs. Mst1-negative breast cancers.

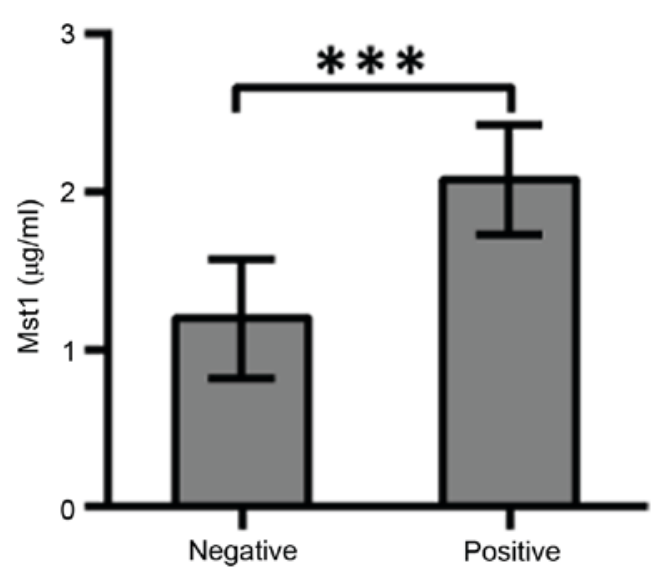

Figure 1. Mst1-detection and measurement by ELISA. Mst1 (C-term) antibodies against plasma antigens were used. An average concentration of $1.8 \mu \mathrm{g} / \mathrm{ml}$ was obtained and used as a discriminative value for Mst1-positive and Mst1-negative patients. ${ }^{* * *} \mathrm{P}<0.001$ vs. negative. Mst1, mammalian sterile 20-like kinase 1.

The IgG level of Mst1-positive vs. Mst1-negative patients was significantly different $(\mathrm{P}<0.0001 ; \mathrm{t}=9.167)$. In total, 85 Mst1-positive and 13 Mst1-negative patients with breast cancer were identified.

Association of Mst1 levels with OS and DFS. To evaluate the significance of Mst1 as a clinical prognostic factor in patients with breast cancer, the two groups of patients were followed up, and the associations between OS, DFS and Mst1 levels were investigated. Patients with positive expression of Mst1 had a significantly improved OS and DFS compared with patients with negative Mst1 expression $(\mathrm{P}<0.0001$; Fig. 2A and B). Univariate Cox analysis indicated that Mst1 positivity had a significant difference in OS in patients with breast cancer $(\mathrm{P}=0.010)$. In multivariate Cox analysis, Mst1 positivity maintained significance as an independent prognostic factor in breast cancer $(\mathrm{P}=0.002$; Table II).

Associations between OS, DFS and clinicopathological features. As expected, OS and DFS were significantly improved in patients with Her2-negative breast cancer (Fig. 2C, $\mathrm{P}=0.0438$; Fig. 2D, $\mathrm{P}=0.0078$ ), lymph node-negative breast cancer (Fig. 2E, $\mathrm{P}=0.0044$; Fig. $2 \mathrm{~F}, \mathrm{P}=0.0379$ ), stage 1 and 2 breast cancer (Fig. 2G, $\mathrm{P}<0.0001$; Fig. $2 \mathrm{H}, \mathrm{P}=0.0001$ ) and tumor size $<2 \mathrm{~cm}$ (Fig. 2I, $\mathrm{P}=0.0019$; Fig. 2J, P=0.0121). Classification of grades and pathological types (ductal vs. all others) did not reveal a prognostic significance (Fig. 3A-D). In addition, no prognostic significance was observed in the comparison of molecular subtypes of breast cancer (luminal vs. others; Fig. 3E and F).

\section{Discussion}

Breast cancer is the most common type of non-cutaneous cancer and the leading cause of cancer-associated mortality among women (1-4). Along with demographical aging and cancer risk factors associated with modern lifestyle, female breast cancer incidence rates demonstrate a rising tendency (5). Screening techniques remain a 

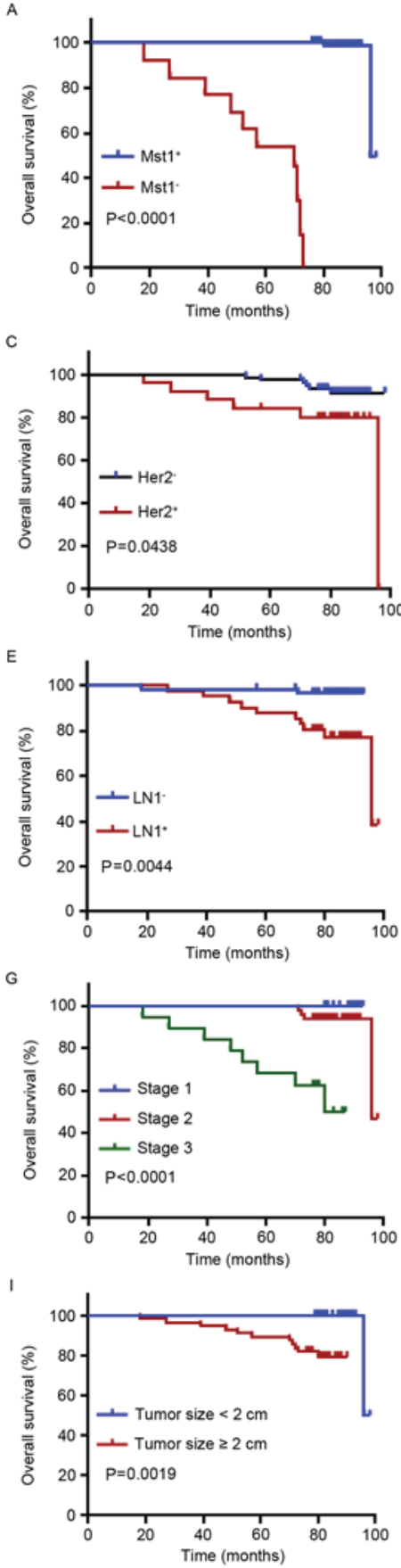
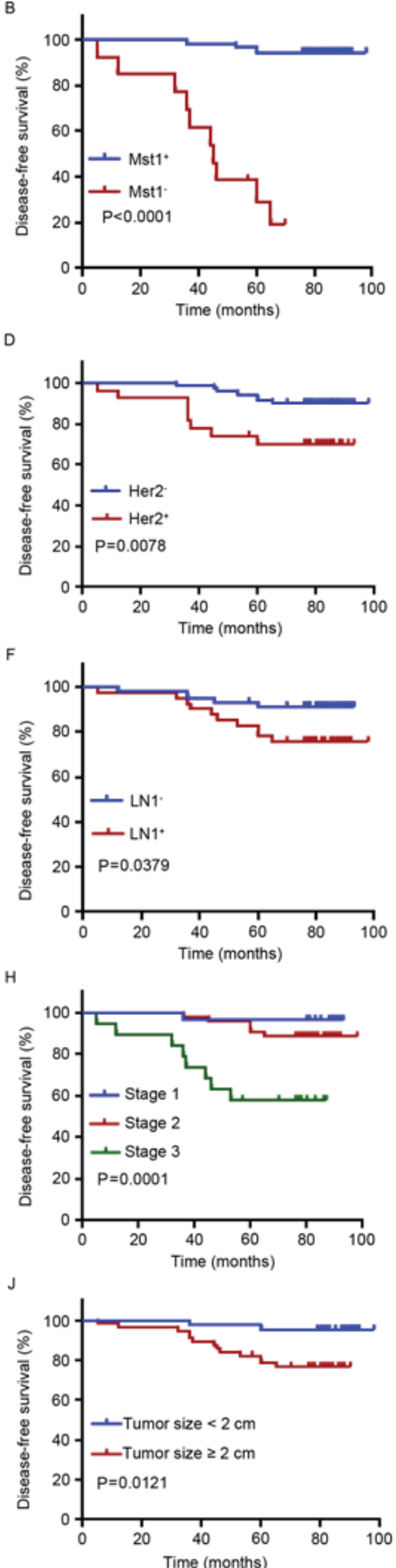

Figure 2. Kaplan-Meier survival analysis curves representing (A) overall and (B) disease free survival according to Mst1 level, (C) overall and (D) disease free survival according to Her2 status, (E) overall and (F) disease free survival according to lymph node status, (G) overall and (H) disease free survival according to tumor stage and (I) overall and (J) disease free survival according to tumor size. Statistical significance is indicated. Mst1, mammalian sterile 20-like kinase 1; Her2, human epidermal growth factor receptor 2; LN, lymph node.

crucial part of prevention and reduction of breast cancer mortality (1).

Diagnostic tumor markers are gaining increasing importance as prognostic and predictive factors (32-34). Current breast cancer markers, including hormone receptor status, tumor-node-metastasisand grading are notsufficient, since breast canceris complex, heterogeneous and alterable (35). Oncologists aim to identify high risk individuals, detect cancer at an early stage, predict outcome, monitor treatment and screen for disease recurrence. During tumor progression, metastasis and anticancer therapy, molecular changes result in various constellations of potential marker proteins $(33,36,37)$. To date, comparatively few markers have been established (38). Therefore, it is crucial to identify easily detectable, non-invasive, novel biological markers with predictive power.

The results of the present study are promising for the use of Mst1 level as an outcome predictor in patients with breast cancer. Mst1 overexpression has been reported to inhibit the growth of human non-small cell lung cancer in vitro and in vivo, reduce intestinal stem cell proliferation and colonic tumorigenesis, inhibit cell proliferation and induce apoptosis 
Table II. Univariate and multivariate analysis of overall survival by the Cox proportional hazards model.

\begin{tabular}{|c|c|c|c|c|}
\hline \multirow[b]{2}{*}{ Clinicopathological variables } & \multicolumn{2}{|c|}{ Univariate analysis } & \multicolumn{2}{|c|}{ Multivariate analysis } \\
\hline & $\mathrm{HR}(95 \% \mathrm{CI})$ & P-value & $\mathrm{HR}(95 \% \mathrm{CI})$ & P-value \\
\hline Age & $0.999(0.922-1.082)$ & 0.982 & $0.696(0.388-1.247)$ & 0.224 \\
\hline Tumor size & $15.987(5.135-49.777)$ & 0.000 & $0.079(0.000-233.758)$ & 0.534 \\
\hline Tumor stage & $12.569(3.514-44.961)$ & 0.000 & $9.002 \times 10^{3}\left(0.000-5.364 \times 10^{34}\right)$ & 0.801 \\
\hline Histological grade & $0.750(0.205-2.743)$ & 0.663 & $0.081(0.001-5.026)$ & 0.233 \\
\hline Lymph node status & $6.811(1.471-31.545)$ & 0.014 & $7.377(0.581-93.673)$ & 0.123 \\
\hline Histological type & $1.768(0.659-4.740)$ & 0.257 & $1.860(0.185-18.743)$ & 0.598 \\
\hline Molecular subtypes & $1.347(0.822-2.207)$ & 0.237 & $7.377(0.581-93.673)$ & 0.239 \\
\hline ER/PR status & $0.757(0.222-2.587)$ & 0.657 & $0.002(0.000-16.843)$ & 0.172 \\
\hline Her2 status & $2.187(0.793-6.033)$ & 0.130 & $5.215 \times 10^{3}\left(0.347-7.837 \times 10^{7}\right)$ & 0.081 \\
\hline Mst1 & $1.157(1.065-1.257)$ & 0.010 & $1.445(1.251-1.670)$ & 0.002 \\
\hline
\end{tabular}

HR, hazard ratio; CI, confidence interval; ER, estrogen receptor; PR, progesterone receptor; Her2, human epidermal growth factor receptor; Mst1, mammalian sterile 20-like kinase 1.
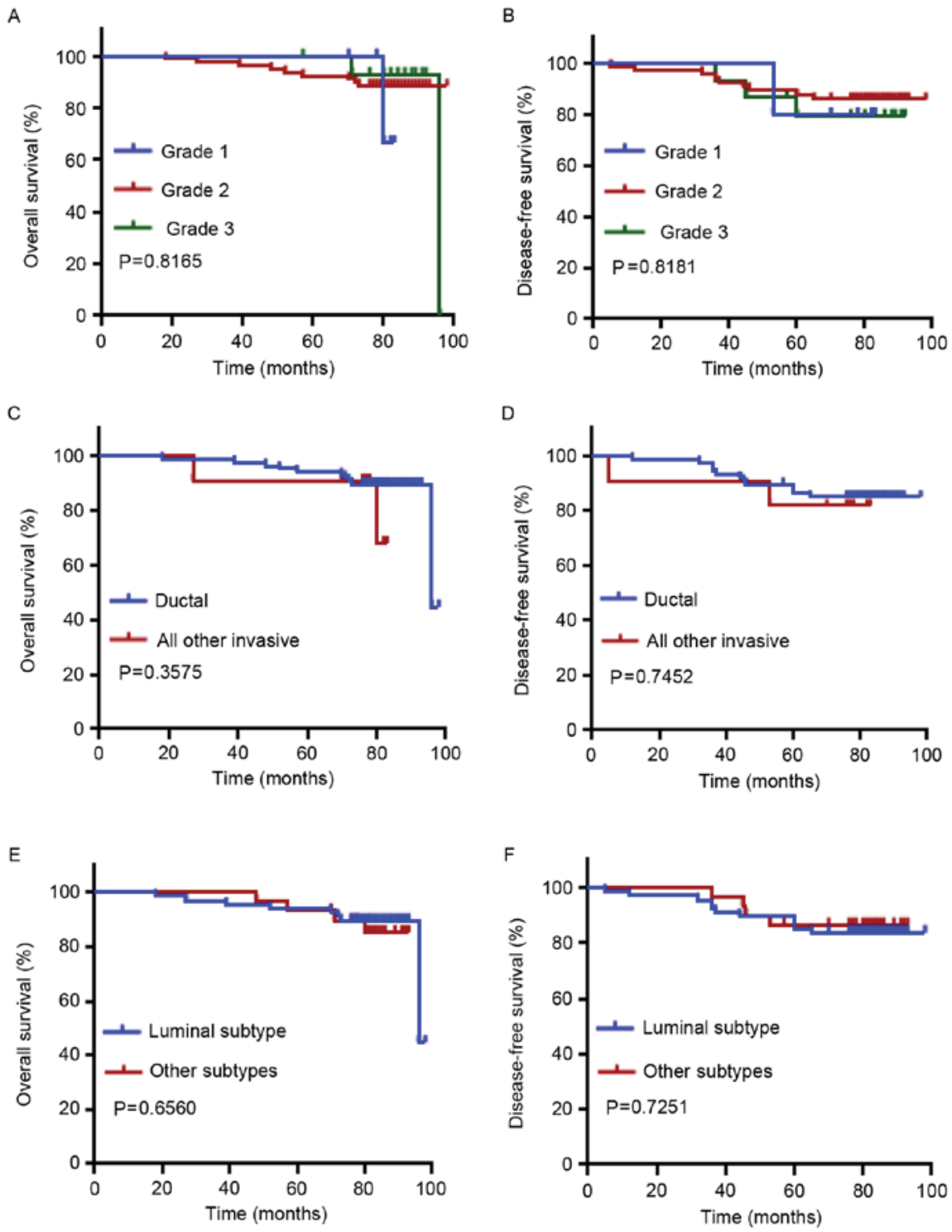

Figure 3. Kaplan-Meier survival analysis curves representing (A) overall and (B) disease free survival according to tumor grade, (C) overall and (D) disease free survival according to pathological type and (E) overall and (F) disease free survival according to subtype. Statistical significance is indicated. 
of HepG2 cells, and induce cisplatin chemosensitivity in hepatocellular carcinoma (20). In colon cancer, nuclear Mst1 expression was associated with tumor grade and shortened survival time (39). Loss of cytoplasmic Mst1 expression is a marker of tumor progression in mismatch-repair-proficient as well as mismatch-repair-deficient colorectal cancers (39). Methylation of the Mst1 promoter is associated with a significantly decreased risk of tumor-associated mortality in patients with soft tissue sarcomas, while alterations of the Mst signaling pathway contribute to poor prognosis (40).

Mst1 is a member of the yeast Ste20-related kinase family and a component of the Ras association domain family member 1-large tumor suppressor kinase 1tumor suppressor network $(41,42)$. Although its physiological function remains to be fully established, it has been proposed as a tumor suppressor protein due to its association with cell proliferation and apoptosis (43). Mst1 is also involved in diverse biological processes, including cellular responses to oxidative stress and longevity (43). Deregulation of these fundamental developmental processes may lead to cancer. The molecular mechanisms are known in Drosophila, where the Hippo signaling pathway controls organ size by restricting mitosis and promoting cell death. In mammals, Mst1, a murine homolog of the Drosophila Hippo, contributes to size control of certain organs, but not all (44).

In the present study, the Mst1 levels of 98 patients with breast cancer with a follow-up period of 98 months were analyzed, and the association of Mst1 levels with survival and clinicopathological characteristics of patients were assessed. In contrast to our previous study (30), a more exact quantification method of ELISA was performed. Using this method obtained objective numeric values (Mst concentration) rather than biased immunohistochemistry-based observations on Mst1 amounts. Additionally, the plasma of patients was used as the detection material. This sampling is easier and more feasible than tumor tissues. To summarize, a novel, easy and effective way to assess Mst1 levels in patients with breast cancer was proposed, which may be further used to predict their prognosis and therapy response.

A cut off was established, and patients were divided into Mst1-positive and Mst1-negative groups. It was revealed that Mst1 positivity was significantly associated with OS, and Mst1-positive patients had an improved OS and DFS compared with Mst1-negative patients. Multivariate analysis also indicated that Mst1 positivity was an independent prognostic factor for breast cancer.

The present, long-term, follow-up study demonstrated that Mst1 expression has prognostic significance in patients with breast cancer and may present potential opportunities for breast cancer therapy in the future.

\section{Acknowledgements}

The present study was supported by the National Key Basic Research Program of China (grant no. 2013CB531601), the National Natural Science Foundation of China (grant no. 30972633), the Scientific Research Foundation for the Returned Overseas Chinese Scholars, State Education Ministry (grant no. 2015-311), the Shanghai Health and Family Planning Commission Project (grant nos. 20134298 and 201640253), the Shanghai Health and Family Planning Commission Fund for Qing Nian Yi Shi Training Project (grant no. 2014118), the Shanghai Yangpu District Science and Technology Commission Project (grant no. 2016-2017), the Shanghai Yangpu District Health and Family Planning Commission Project (grant nos. 2011-2013 and 2016-2017), the Shanghai Yangpu District Health and Family Planning Commission Fund for Bai Yi Deng Gao Training Project (grant no. 2014-2016) and the Academic Leader in Climbing Program from Yang-Pu Center Hospital (grant no. YE2201608).

\section{References}

1. Siegel RL, Miller KD and Jemal A: Cancer statistics, 2015. CA Cancer J Clin 65: 5-29, 2015.

2. Torre LA, Bray F, Siegel RL, Ferlay J, Lortet-Tieulent J and Jemal A: Global cancer statistics, 2012. CA Cancer J Clin 65: 87-108, 2015.

3. Siegel RL, Miller KD and Jemal A: Cancer statistics, 2016. CA Cancer J Clin 66: 7-30, 2016.

4. Swanton C, Burrell RA and Futreal PA: Breast cancer genome heterogeneity: A challenge to personalised medicine? Breast Cancer Res 13: 104, 2011.

5. Jackson SE and Chester JD: Personalised cancer medicine. Int J Cancer 137: 262-266, 2015.

6. Tyrer J, Duffy SW and Cuzick J: A breast cancer prediction model incorporating familial and personal risk factors. Stat Med 23: 1111-1130, 2004.

7. Yersal O and Barutca S: Biological subtypes of breast cancer: Prognostic and therapeutic implications. World J Clin Oncol 5: 412-424, 2014.

8. Garg M, Nagpal N, Sidhu DS and Singh A: Effect of lump size and nodal status on prognosis in invasive breast cancer: Experience from Rural India. J Clin Diagn Res 10: PC08-PC11, 2016.

9. Senkus E, Cardoso F and Pagani O: Time for more optimism in metastatic breast cancer? Cancer Treat Rev 40: 220-228, 2014.

10. Marmé F and Schneeweiss A: Personalised therapy in breast cancer. Onkologie 35 (Suppl 1): S28-S33, 2012.

11. Andersen SL, Terry DF, Wilcox MA, Babineau T, Malek K and Perls TT: Cancer in the oldest old. Mech Ageing Dev 126: 263-267, 2005.

12. Lichtman SM: Guidelines for the treatment of elderly cancer patients. Cancer Control 10: 445-453, 2003

13. Baird RD and Caldas C: Genetic heterogeneity in breast cancer: The road to personalized medicine? BMC Med 11: 151, 2013.

14. Sabatier R, Gonçalves A and Bertucci F: Personalized medicine: Present and future of breast cancer management. Crit Rev Oncol Hematol 91: 223-233, 2014.

15. Dawson SJ, Rueda OM, Aparicio S and Caldas C: A new genome-driven integrated classification of breast cancer and its implications. EMBO J 32: 617-628, 2013.

16. Ellsworth RE, Decewicz DJ, Shriver CD and Ellsworth DL: Breast cancer in the personal genomics era. Curr Genomics 11: 146-161, 2010.

17. Dancey JE, Bedard PL, Onetto N and Hudson TJ: The genetic basis for cancer treatment decisions. Cell 148: 409-420, 2012.

18. André F, Bachelot T, Commo F, Campone M, Arnedos M, Dieras V, Lacroix-Triki M, Lacroix L, Cohen P, Gentien D, et al: Comparative genomic hybridisation array and DNA sequencing to direct treatment of metastatic breast cancer: A multicentre, prospective trial (SAFIR01/UNICANCER). Lancet Oncol 15: 267-274, 2014.

19. Song H, Mak KK, Topol L, Yun K, Hu J, Garrett L, Chen Y, Park O, Chang J, Simpson RM, et al: Mammalian Mst1 and Mst2 kinases play essential roles in organ size control and tumor suppression. Proc Natl Acad Sci USA 107: 1431-1436, 2010.

20. Qin F, Tian J, Zhou D and Chen L: Mst1 and Mst2 kinases: Regulations and diseases. Cell Biosci 3: 31, 2013.

21. Cinar B, Collak FK, Lopez D, Akgul S, Mukhopadhyay NK, Kilicarslan M, Gioeli DG and Freeman MR: MST1 is a multifunctional caspase-independent inhibitor of androgenic signaling. Cancer Res 71: 4303-4313, 2011. 
22. Abdollahpour H, Appaswamy G, Kotlarz D, Diestelhorst J, Beier R, Schäffer AA, Gertz EM, Schambach A, Kreipe HH, Pfeifer D, et al: The phenotype of human STK4 deficiency. Blood 119: 3450-3457, 2012.

23. Praskova M, Khoklatchev A, Ortiz-Vega S and Avruch J: Regulation of the MST1 kinase by autophosphorylation, by the growth inhibitory proteins, RASSF1 and NORE1, and by Ras. Biochem J 381: 453-462, 2004.

24. Graves JD, Gotoh Y, Draves KE, Ambrose D, Han DK, Wright M, Chernoff J, Clark EA and Krebs EG: Caspase-mediated activation and induction of apoptosis by the mammalian Ste20-like kinase Mst1. EMBO J 17: 2224-2234, 1998.

25. Qin F, Tian J, Zhou D and Chen L: Mst1 and Mst2 kinases: Regulations and diseases. Cell Biosci 3: 31, 2013.

26. Rawat SJ, Creasy CL, Peterson JR and Chernoff J: The tumor suppressor Mstl promotes changes in the cellular redox state by phosphorylation and inactivation of peroxiredoxin-1 protein. J Biol Chem 288: 8762-8771, 2013.

27. Guo C,Zhang $X$ and Pfeifer GP: The tumor suppressor RASSF1A prevents dephosphorylation of the mammalian STE20-like kinases MST1 and MST2. J Biol Chem 286: 6253-6261, 2011.

28. Warfel NA and Newton AC: Pleckstrin homology domain leucine-rich repeat protein phosphatase (PHLPP): A new player in cell signaling. J Biol Chem 287: 3610-3616, 2012.

29. Artemenko Y, Batsios P, Borleis J, Gagnon Z, Lee J, Rohlfs M, Sanséau D, Willard SS, Schleicher M and Devreotes PN: Tumor suppressor Hippo/MST1 kinase mediates chemotaxis by regulating spreading and adhesion. Proc Natl Acad Sci USA 109: 13632-13637, 2012.

30. Lin X, Cai F, Li X, Kong X, Xu C, Zuo X and Yang Q: Prognostic significance of mammalian sterile 20-like kinase 1 in breast cancer. Tumour Biol 34: 3239-3243, 2013.

31. Pan X, Zeng SL, Yu DH, Liang XL, Ji CD, Pan BJ, Cai JL, Wang Y, Min Y, Fang W and Liao WQ: Variable domain of the heavy chain of heavy-chain antibody of the Rv0733 antigen of mycobacterium tuberculosis panned and identified from a nonimmune llama VHH phage display library. Int J Clin Exp Pathol 9: 2869-2878, 2016.

32. Yoneda A, Lendorf ME, Couchman JR and Multhaupt HA: Breast and ovarian cancers: A survey and possible roles for the cell surface heparan sulfate proteoglycans. J Histochem Cytochem 60: 9-21, 2012.
33. Banin Hirata BK, Oda JM, Losi Guembarovski R, Ariza CB, de Oliveira CE and Watanabe MA: Molecular markers for breast cancer: Prediction on tumor behavior. Dis Markers 2014: 513158, 2014.

34. Esteva FJ and Hortobagyi GN: Prognostic molecular markers in early breast cancer. Breast Cancer Res 6: 109-118, 2004.

35. Prat A, Parker JS, Karginova O, Fan C, Livasy C, Herschkowitz JI, He X and Perou CM: Phenotypic and molecular characterization of the claudin-low intrinsic subtype of breast cancer. Breast Cancer Res 12: R68, 2010.

36. Kumar R: Breast cancer tumor markers. J Solid Tumors 2: 43, 2012.

37. Payne SJ, Bowen RL, Jones JL and Wells CA: Predictive markers in breast cancer-the present. Histopathology 52: 82-90, 2008.

38. Penault-Llorca F and Viale G: Pathological and molecular diagnosis of triple-negative breast cancer: A clinical perspective. Ann Oncol 23 (Suppl 6): vi19-vi22, 2012.

39. Minoo P, Zlobec I, Baker K, Tornillo L, Terracciano L, Jass JR and Lugli A: Prognostic significance of mammalian sterile 20-like kinase 1 in colorectal cancer. Mod Pathol 20: 331-338, 2007.

40. Seidel C, Schagdarsurengin U, Blümke K, Würl P, Pfeifer GP, Hauptmann S, Taubert H and Dammann R: Frequent hypermethylation of MST1 and MST2 in soft tissue sarcoma. Mol Carcinog 46: 865-871, 2007.

41. Cinar B, Collak FK, Lopez D, Akgul S, Mukhopadhyay NK, Kilicarslan M, Gioeli DG and Freeman MR: MST1 is a multifunctional caspase-independent inhibitor of androgenic signaling. Cancer Res 71: 4303-4313, 2011.

42. Oh HJ, Lee KK, Song SJ, Jin MS, Song MS, Lee JH, Im CR, Lee JO, Yonehara S and Lim DS: Role of the tumor suppressor RASSF1A in Mst1-mediated apoptosis. Cancer Res 66: 2562-2569, 2006

43. Lehtinen MK, Yuan Z, Boag PR, Yang Y, Villén J, Becker EB, DiBacco S, de la Iglesia N, Gygi S, Blackwell TK and Bonni A: A conserved MST-FOXO signaling pathway mediates oxidative-stress responses and extends life span. Cell 125: 987-1001, 2006.

44. Avruch J, Zhou D, Fitamant J, Bardeesy N, Mou F and Barrufet LR: Protein kinases of the Hippo pathway: Regulation and substrates. Semin Cell Dev Biol 23: 770-784, 2012. 